\title{
Direct Measurement of Large, Diffuse, Optical Structures
}

\author{
Babak N. Saif \\ Space Telescope Science Institute, Baltimore, Maryland 21219 U.S.A \\ Ritva Keski-Kuha \& Lee Feinberg \\ NASA Goddard Space Flight Center, Greenbelt, Maryland 20770 U.S.A \\ J.C. Wyant \\ University of Arizona, Optical Sciences Center, Tucson, Arizona 85701 U.S.A \\ C. Atkinson \\ Northrop Grumman, Los Angeles, CA U.S.A
}

\begin{abstract}
Digital Speckle Pattern Interferometry (DSPI) is a well-established method for the measurement of diffuse objects in experimental mechanics. DSPIs are phase shifting interferometers. Three or four bucket temporal phase shifting algorithms are commonly used to provide phase shifting. These algorithms are sensitive to vibrations and can not be used to measure large optical structures far away from the interferometer. In this research a simultaneous phase shifted interferometer, PhaseCam product of 4D Technology Corporation in Tucson Arizona, is modified to be a Simultaneous phase shifted Digital Speckle Pattern Interferometer (SDSPI). Repeatability, dynamic range, and accuracy of the SDSPI are characterized by measuring a $5 \mathrm{~cm} \times 5 \mathrm{~cm}$ carbon fiber coupon.
\end{abstract}

Keywords: DSPI, Simultaneous phase shifting, Experimental mechanics

\section{INTRODUCTION}

Upcoming astronomy and astrophysical missions are James Webb Space Telescope (JWST), and Terrestrial Planet Finder (TPF). JWST is a cryogenic segmented telescope with primary mirror diameters of 6.5 meter. TPF could be a JWST telescope with a coronagraph or it could be a large baseline stellar interferometer.

Large optics and large optical structures to support the optics are common among these missions. Stability of the optics and the optical structures are critical for all these missions. Recent simultaneous phase shifted interferometers enable the measurements of specular surfaces to the required accuracies at cryogenic chambers but required accuracies for measurements of the non-specular surfaces are challenging. The optical structures are generally made of diffuse materials such as reinforced composite material. There are two methods for measurements of diffuse surfaces. One is to populate the optical structures with optical metrologies such as small mirrors, retro reflectors and use distance measuring interferometers to measure the stability of the structures by measuring the stability of the attached metrology. Required stability accuracies for the new missions make it hard to distinguish the stability of the structures from stability of the attached metrology. For example the stability of the primary mirror back plane of JWST is a major concern in co-phasing of the telescope and long term stability of its image quality. The back plane stability requirement in the out of plane motion is $15 \mathrm{~nm}$ RMS. The second way is to use speckle metrology such as DSPI, and Digital Speckle Photography as direct ways to measure the structures.

A direct method is needed to measure large cryogenic structures with the required accuracies. The technology that is being developed here potentially provides us with an interferometer that has the required accuracies for these measurements. 


\section{DSPI}

Operation of a first CW He-Ne in 1960 revealed a granular pattern called Speckle. In 1970s [1-2] first speckle interferometry papers were published. In 1975 photographic film was replaced by TV [3], [4] but the low resolution of TV was not appealing. The field was not active till the mid 80 s when the phase shifting speckle interferometry came about [5]. Advances in computers, algorithms, frame grabbers, and phase shifting methods in 1990s till now made speckle interferometry an industrial tool. Today miniaturize hand held 3D temporal phase shifted DSPIs allows 3.D measurements from close proximity with accuracies for out of plane motions of $20 \mathrm{~nm}$ RMS, and $26 \mathrm{~nm}$ RMS for the in-plane motions [6]. Also DSPIs that are based on temporal phase shifted shear interferometers have accuracies of about $60 \mathrm{~nm}$ RMS [7], [8].

The main topic of this research is to characterize and design a DSPI based on simultaneous phase shifting interferometry. The temporal phase shifting is sensitive to vibration and prevents measurements of large structures. The outcome of this work is a Simultaneous phase shifted DSPI (SDSPI) that remotely enables measurements of large cryogenic structures with accuracy of tens of nm RMS.

\section{SAMPLES}

The optical structures are made of composite material called carbon fiber. The material is black and diffuse. There are two kinds of finish to the carbon fiber. One has more specular reflection (shiny side) than the other (matte side). Reflectivity of samples with both finishes is measured using a spectrophotometer with integrating sphere. The reflectivity is about $7-8 \%$ at 0.633 -micron wavelength over half of the hemisphere. Figure 1 . shows the reflectivity of both finishes as function of the wavelength.

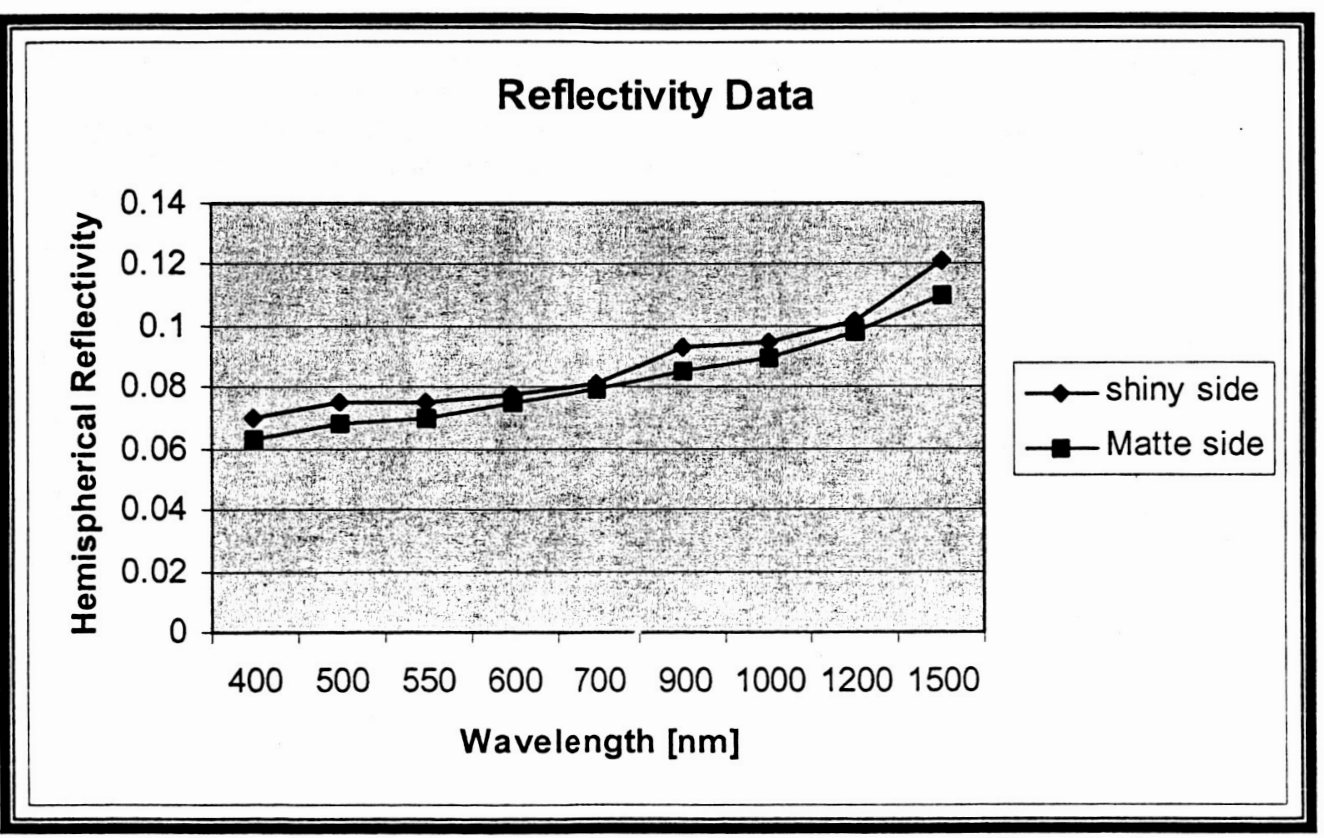

FIGURE 1. The reflectivity of the carbon fiber material with two different finishes 


\section{ACCURACY MEASUREMENTS}

In order to do accuracy measurements, a $5 \mathrm{~cm} \times 5 \mathrm{~cm}$ flat mirror is acquired. A $2.5 \mathrm{~cm} \times 5 \mathrm{~cm}$ carbon fiber coupon is glued on top of the flat mirror; Figure 2. The mirror and the carbon fiber assembly are mounted on a rotating stage. The optical set up to measure accuracy is shown in Figure.2 and Figure.3. The mirror/carbon fiber is $2.5-5$ $\mathrm{cm}$ from the interferometer in the collimated space. An attenuator is used to equalize the light from the mirror and the carbon fiber sections. The beam ratio between the test beam, from mirror and carbon fiber, and reference beam is optimized to get good contrast fringes. An interferogram of the test beam and the reference beam is acquired. This interferogram is the reference or the baseline measurement. The sample is rotated and another interferogram is acquired. The phase is calculated; equation 1. Figure 4a, shows the tilt fringes due to rotation of the object. Fringes on the top are the fringes measured over the carbon fiber material with shiny finish. At the bottom are the fringes measured on the flat specular mirror section. More tilt is introduced and the procedure is repeated; Figure 4.b. The subtraction is done from the baseline measurement.

$$
\phi_{f}-\phi_{i}=\operatorname{ArcTan}\left(\frac{I_{f, 2}-I_{f, 4}}{I_{f, 3}-I_{f, 1}}\right)-\operatorname{ArcTan}\left(\frac{I_{i, 2}-I_{i, 4}}{I_{i, 3}-I_{i, 1}}\right)
$$

Where $I_{i, 1}$ and $I_{f, 1}$ are object and modified object interferograms with a zero phase shift.

$I_{i, 2}$ and $I_{f, 2}$ are object and modified object interferograms with a $\frac{\pi}{2}$ phase shift.

$I_{i, 3}$ and $I_{f, 3}$ are object and modified object with $\pi$ phase shift.

$I_{i, 4}$ and $I_{f, 4}$ are object and modified object interferograms with $\frac{3 \pi}{2}$ phase shift.

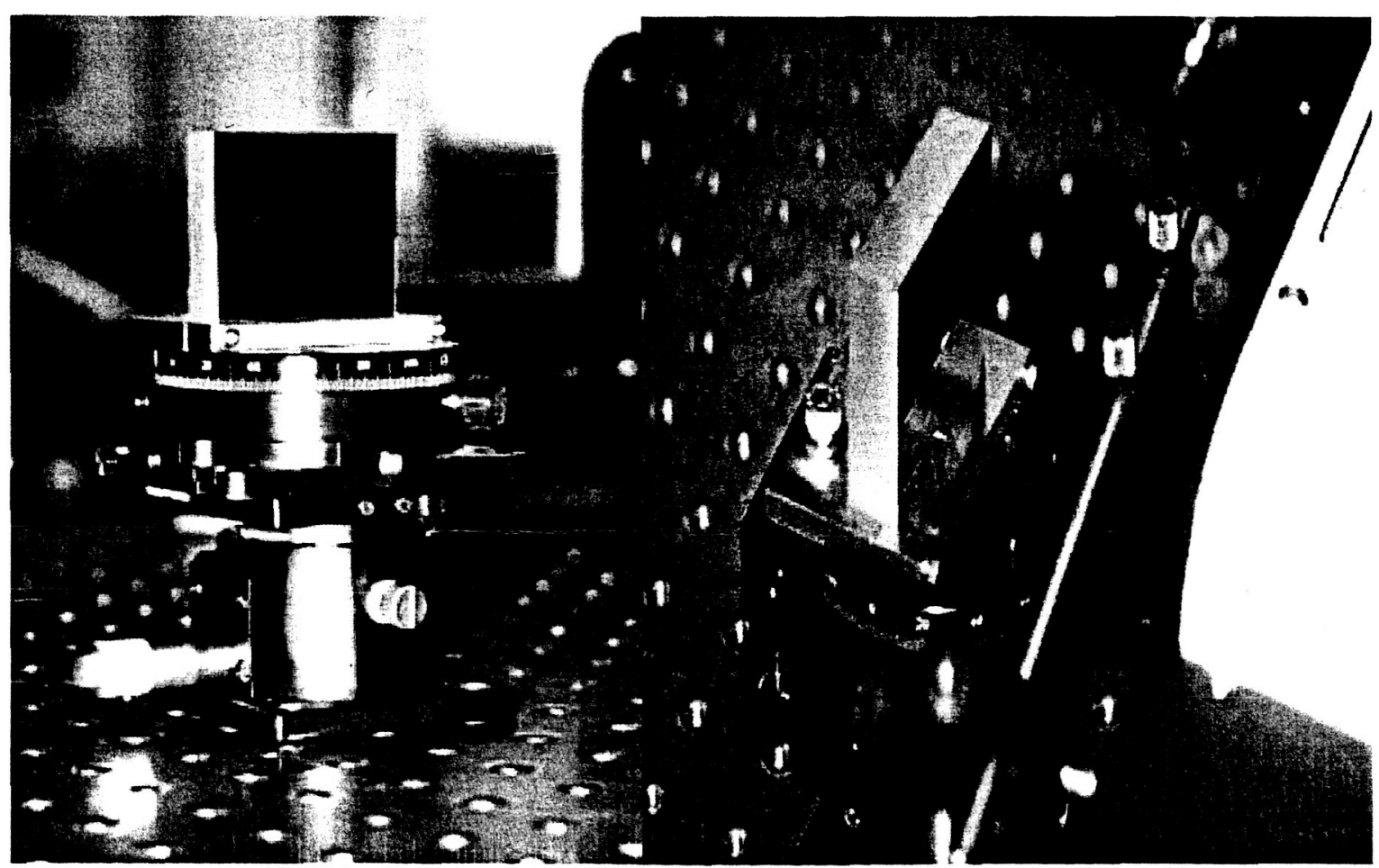

FIGURE 2. Flat mirror with carbon fiber coupon at the collimated space of PhaseCam 


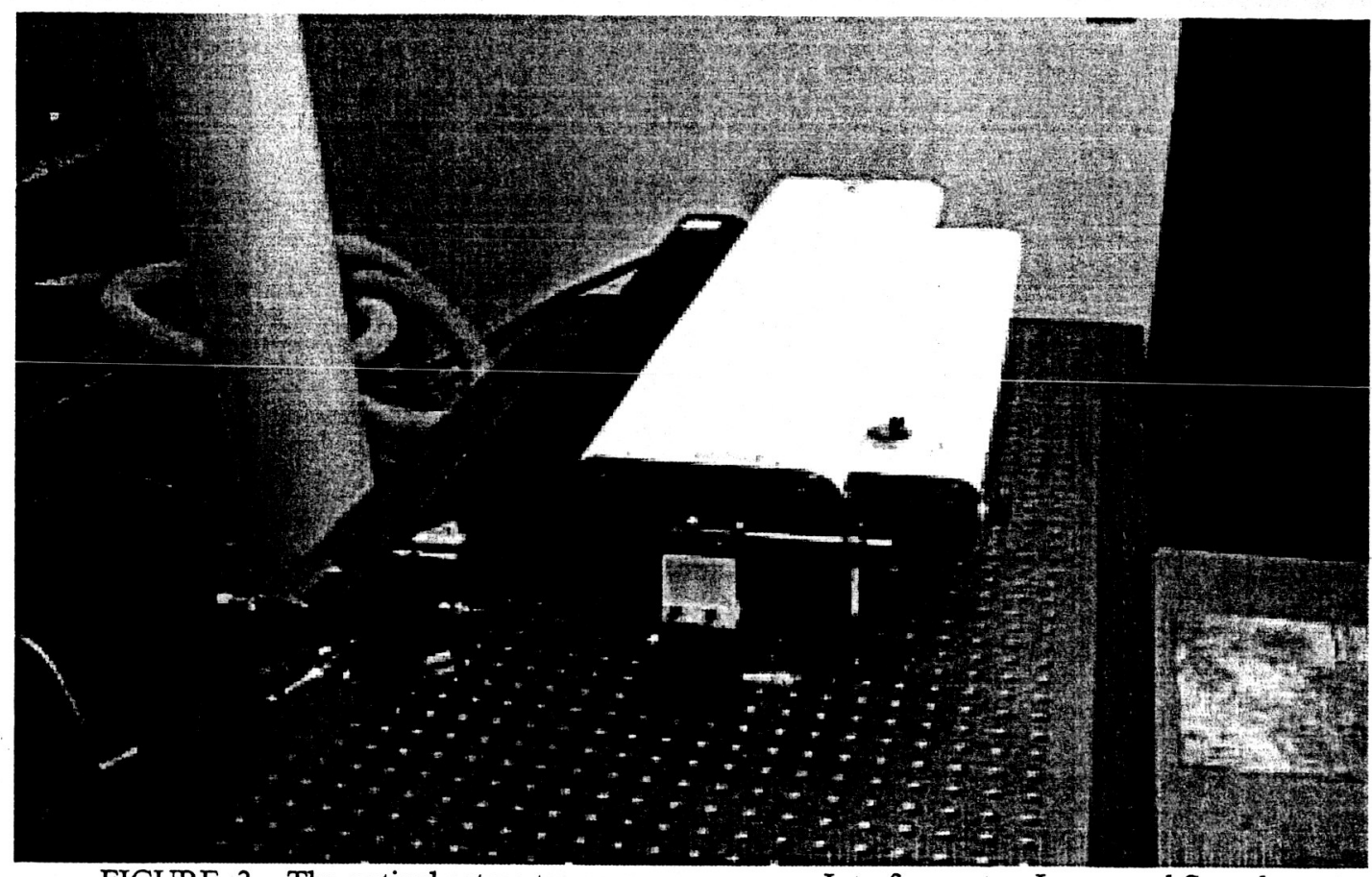

FIGURE .3. The optical set up to measure accuracy: Interferometer, Laser, and Sample
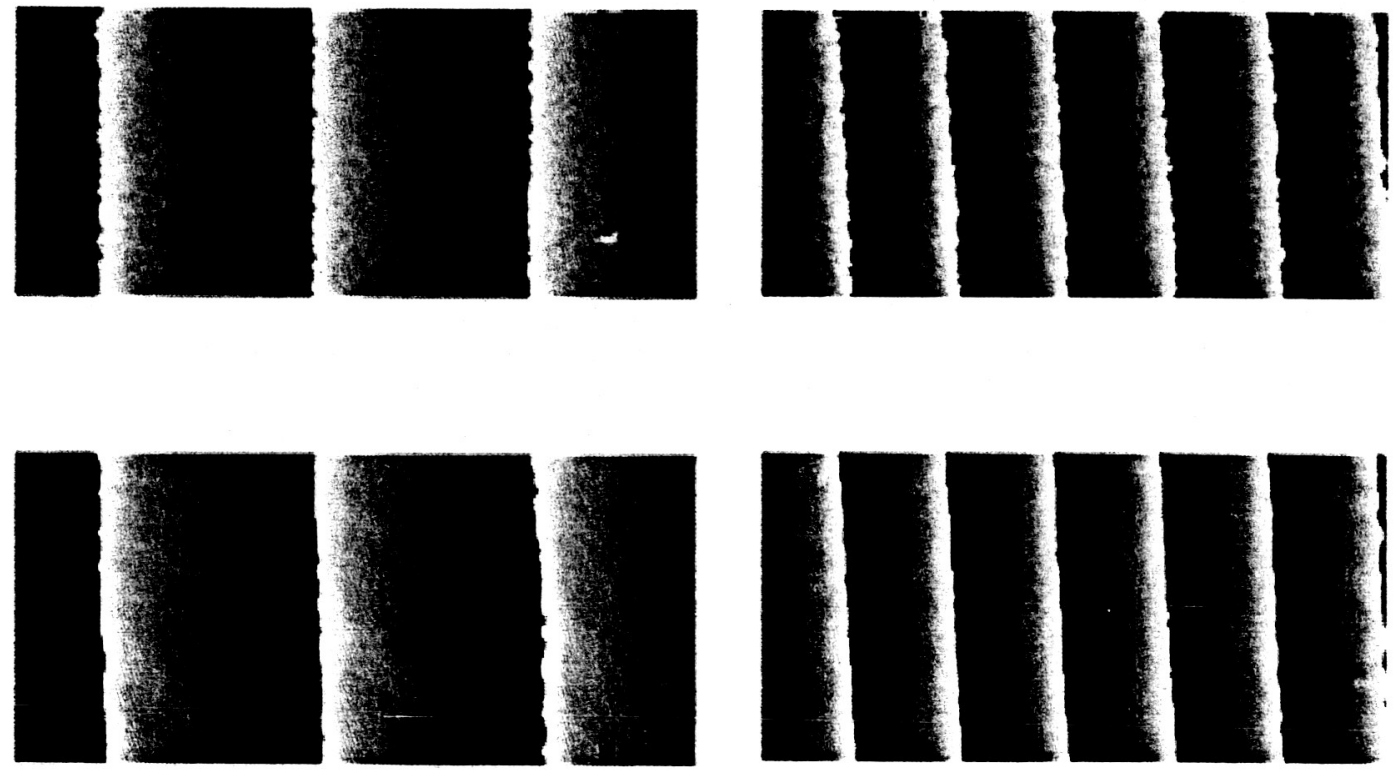

FIGURE. 4 a \& b

Top: Fringes from carbon fiber

Bottom: Fringes from specular flat mirror 
Averaging of $5 \times 5$ pixels are used to smooth out the speckle noise for the wrapped phase. Next the data is unwrapped using Flynn's algorithm. Using 4Sight software product of 4D Technology, the piston between the carbon fiber coupon and the flat mirror is zeroed out.

Now that the data for carbon fiber and mirror are on the same plane, tilt is removed from that plane using the 4Sight software. The residual RMS errors on the mirror side and the carbon fiber side are measured.

The residual RMS for the mirror is 0.0085 waves. The residual for the carbon fiber is 0.015 waves. Measurement is repeated many times and the summary of the result is in Table.1.

\begin{tabular}{|c|c|c|c|}
\hline $\begin{array}{c}\text { Number of } \\
\text { Measurements }\end{array}$ & Mirror RMS & Backplane RMS & Integration Time \\
\hline 1 & $.0063[\mathrm{wv}]$ & $.0228[\mathrm{wv}]$ & $.43[\mathrm{~ms}]$ \\
\hline 2 & $.0058[\mathrm{wv}]$ & $.0187[\mathrm{wv}]$ & $.43[\mathrm{~ms}]$ \\
\hline 3 & $.0053[\mathrm{wv}]$ & $.0180[\mathrm{wv}]$ & $.43[\mathrm{~ms}]$ \\
\hline 4 & $.0076[\mathrm{wv}]$ & $.0182[\mathrm{wv}]$ & $.43[\mathrm{~ms}]$ \\
\hline 5 & $.0061[\mathrm{wv}]$ & $.0167[\mathrm{wv}]$ & $.43[\mathrm{~ms}]$ \\
\hline 6 & $.0085[\mathrm{wv}]$ & $.0150[\mathrm{wv}]$ & $1[\mathrm{~ms}]$ \\
\hline 7 & $.0089[\mathrm{wv}]$ & $.0153[\mathrm{wv}]$ & $1[\mathrm{~ms}]$ \\
\hline 8 & $.0097[\mathrm{wv}]$ & $.0185[\mathrm{wv}]$ & $1[\mathrm{~ms}]$ \\
\hline & & & \\
\hline
\end{tabular}

TABLE 1. Summary of the measurements of carbon fiber (shiny) and mirror

On the average the Residual RMS for the mirror is about .0073 waves $(4.6 \mathrm{~nm})$ and for the carbon fiber is about .018 waves $(11 \mathrm{~nm})$.

Similar measurements are done on a carbon fiber coupon with matte finish. Figure $.5 \mathrm{a} \& \mathrm{~b}$ shows the fringes for the matte finish and a flat mirror. Carbon fiber coupon is on the top again. The optical set up is identical to what is described above. Table. 2 is the summary of the measurements for the matte side and the mirror.
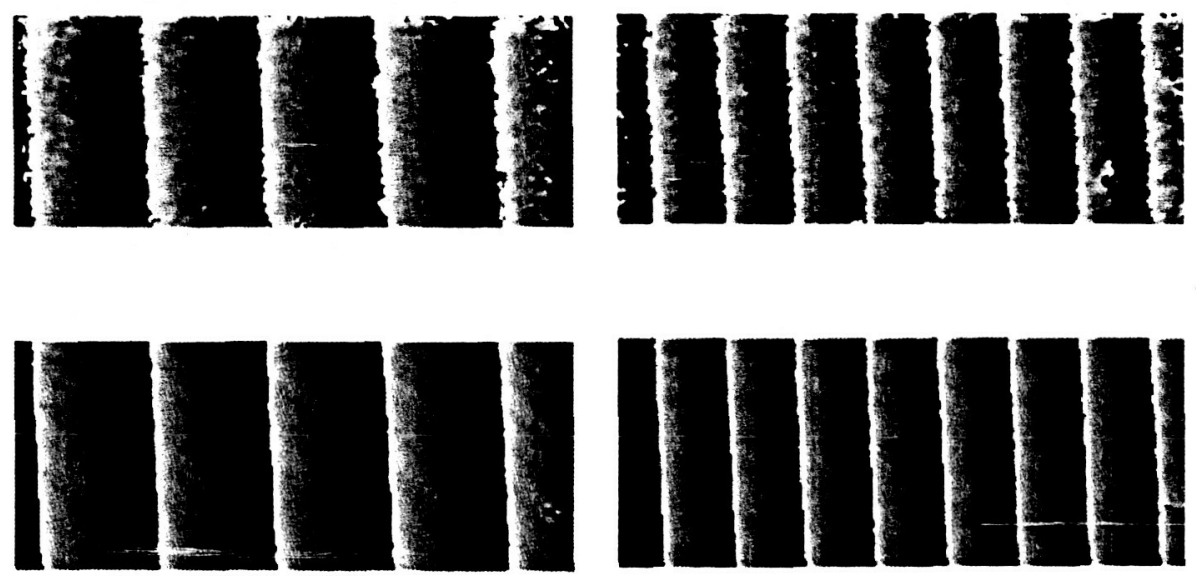

FIGURE 5 a\&b Fringes of carbon fiber (matte) on the top and the mirror on the bottom

5

American Institute of Aeronautics and Astronautics 


\begin{tabular}{|c|c|c|c|}
\hline $\begin{array}{c}\text { Number of } \\
\text { Measurements }\end{array}$ & Mirror RMS & Backplane RMS & $\begin{array}{c}\text { Integration } \\
\text { Time }\end{array}$ \\
\hline 1 & $.0081[\mathrm{wv}]$ & $.0535[\mathrm{wv}]$ & $1[\mathrm{~ms}]$ \\
\hline 2 & $.0088[\mathrm{wv}]$ & $.0313[\mathrm{wv}]$ & $1[\mathrm{~ms}]$ \\
\hline 3 & $.0089[\mathrm{wv}]$ & $.0419[\mathrm{wv}]$ & $1[\mathrm{~ms}]$ \\
\hline 4 & $.0087[\mathrm{wv}]$ & $.0369[\mathrm{wv}]$ & $1[\mathrm{~ms}]$ \\
\hline 5 & $.0093[\mathrm{wv}]$ & $.0464[\mathrm{wv}]$ & $1[\mathrm{~ms}]$ \\
\hline
\end{tabular}

TABLE 2. Summary of the residual errors for carbon fiber with matte finish and mirror

Average RMS residual for the mirror is like before about $5.54 \mathrm{~nm}$ and for the matte side of the carbon fiber is about $27 \mathrm{~nm}$. Comparing $27 \mathrm{~nm}$ RMS with $11 \mathrm{~nm}$ RMS, for the carbon fiber with shiny finish, confirms that accuracy is surface dependent. The rougher the surface, the higher the speckle noise, the smaller the collected power of the test beam, and the larger the phase error. It should be noted that fringes are formed for carbon fiber with shiny finish even with integration of $.4 \mathrm{~ms}$. Fringes are not formed for carbon fiber with matte finish with integration less than a milli-second. To form fringes with good visibility $4-5\left[\mathrm{~nJ} / \mathrm{mm}^{\wedge} 2\right]$ is required on the CCD. The higher the power in the test beam, the less integration time is needed on the detector. Minimizing the integration time, results in less sensitivity to vibration, which is crucial for measuring large structures in the cryogenic chambers.

The measurements above are all single shot measurements. No averaging is done by adding several measurements together. Accuracies above can be improved by averaging many measurements over a time period provided that the vibration would not corrupt the data in this period. 


\section{REPEATABILITY MEASUREMENT}

The optical set up is the same as the set up for accuracy measurements. A carbon fiber coupon with a matte finish is set in front of the PhaseCam at the collimated space. An initial interferogram of the coupon is taken and stored as the base line. The coupon is tilted using the rotating stage. The phase difference is calculated as before. RMS value for this measurement is recorded. Without tilting the coupon, ten more measurements are done. RMS for each measurement is recorded. The repeatability of the interferometer is the standard deviation of the recorded RMS measurements. Summary of the result is in Table.3. Repeatability of the measurements is $.23 \mathrm{~nm}$ RMS.

\begin{tabular}{|c|c|c|}
\hline Number of Measurements & P-V [micron] & RMS [micron] \\
\hline 1 & .2974 & .0104 \\
\hline 2 & .2224 & .0105 \\
\hline 3 & .2418 & .0103 \\
\hline 4 & .2607 & .0102 \\
\hline 5 & .3176 & .0101 \\
\hline 6 & .2602 & .0101 \\
\hline 7 & .2079 & .0098 \\
\hline 8 & .2135 & .0102 \\
\hline 9 & .3070 & .0100 \\
\hline 10 & .2115 & .0099 \\
\hline 11 & .2205 & .0101 \\
\hline Average & .2509 & $.2324[\mathrm{~nm}]$ \\
\hline Standard Deviation & .1288 & \\
\hline
\end{tabular}

TABLE 3. Summary of repeatability measurements 


\section{DYNAMIC RANGE MEASUREMENT}

Dynamic range of an interferometer is the maximum tilt of the object that can be measured at the collimating space with no diverging lens. To do this a $2.5 \mathrm{~cm} \times 2.5 \mathrm{~cm}$ piece of carbon fiber with matte finish is placed right in front of the interferometer at the collimating space. The beam size on the sample is about 7 millimeter in diameter. The sample is mounted on a rotational stage. An interferogram of the object is acquired without any tilt, Figure. 6. This is our reference or baseline for our subsequent measurements. Small amount of tilt is introduced and the reference phase is subtracted. Set of fringes in Figure .7. is the tilt of the object.

Next, more tilt is introduced and reference is subtracted. This process of incremental addition of tilt is continued till fringes are not resolved; Figure .8

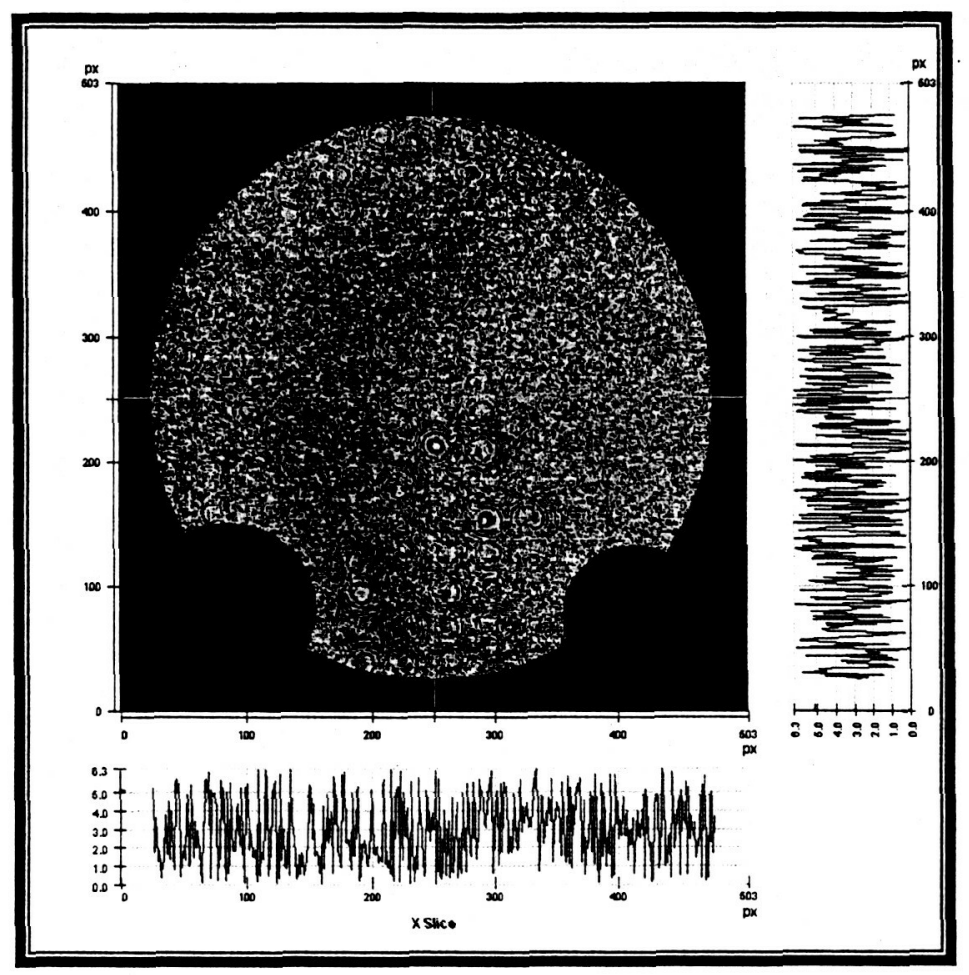

FIGURE 6. The interferogram of carbon fiber (matte) 


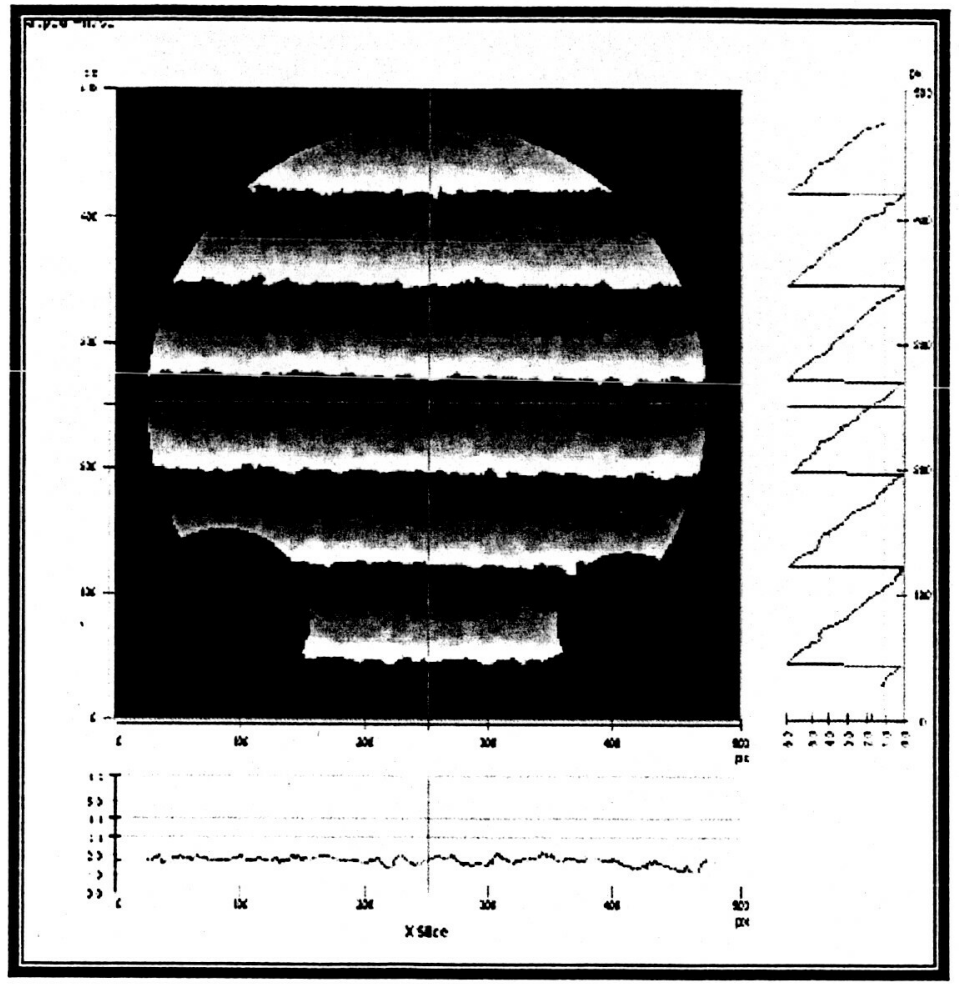

FIGURE 7. The tilt interferogram for carbon fiber (matte) 


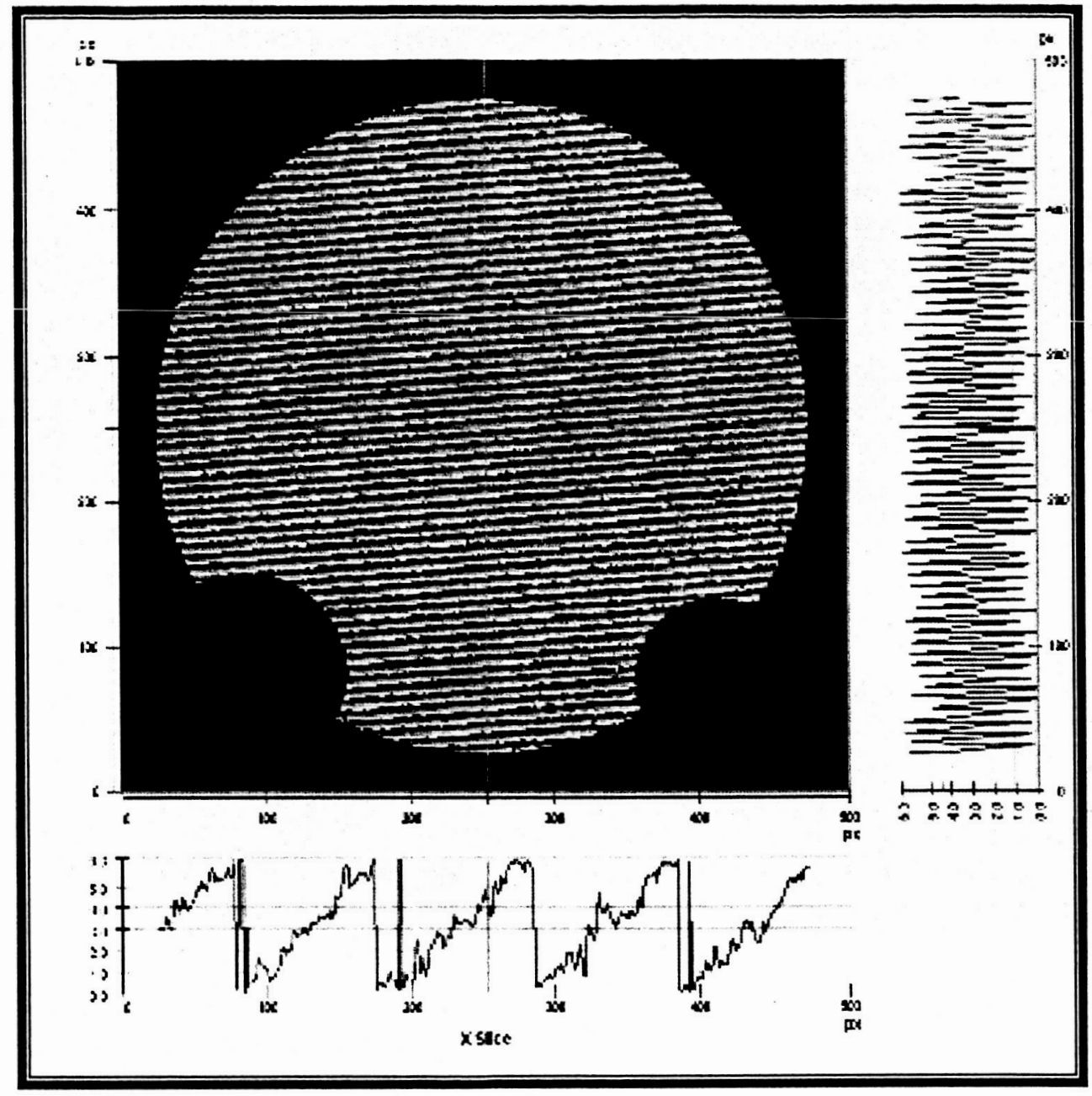

FIGURE. 8. The maximum number of tilt, dynamic range

The maximum number of the tilt fringes is 58 waves, which is 36.7 micron on the wavefront and 18.4 micron on the object. Dynamic range of the interferometer in measuring carbon fiber material with matte finish is 18.4 micron. Using the last interferogram as a new baseline, another 18.4 micron of tilt can be added before updating the baseline again.

The characterization of the speckle interferometer is done in the same way as any classical interferometer is characterized. All the characterization is at the collimating space with no diverging lens and measuring only tilts. Accuracy, repeatability, and dynamic range of the speckle interferometer are measured.

\section{A. DEFORMATION MEASUREMENT}

To deform a carbon fiber coupon $(5 \mathrm{~cm} \times 5 \mathrm{~cm})$, a holder is designed to hold the coupon on two sides, Figure 9 a. A high precision micrometer is used as a plunger to push the coupon from the backside with 0.5 -micron increments; Figure 9b. 


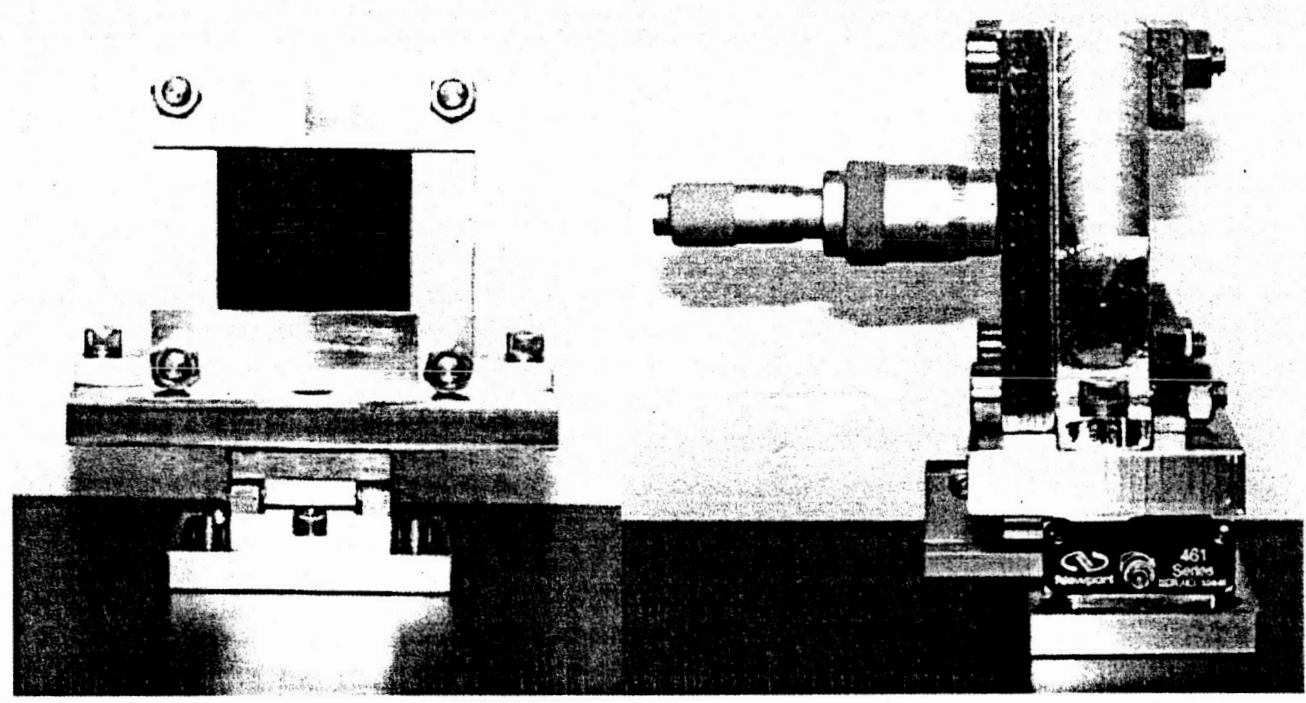

FIGURE 9. $a \& b$ Holder for the carbon fiber coupon

The optical setup is a typical setup to measure concave mirrors in the test arm of an interferometer; Figure 10. A diverging lens is used to illuminate the coupon. The same lens images the coupon to 1-2 inches from the front of the interferometer. The size of the image is 7 millimeter. To measure deformation over the sample $(5 \mathrm{~cm} \mathrm{x} 5 \mathrm{~cm})$, a more powerful laser is required. In deformation measurements the forward power in the test beam is $15 \mathrm{mw}$. The F\# of the lens is adjusted to illuminate the whole holder. The optical set up is shown in Figure 10 and 11.

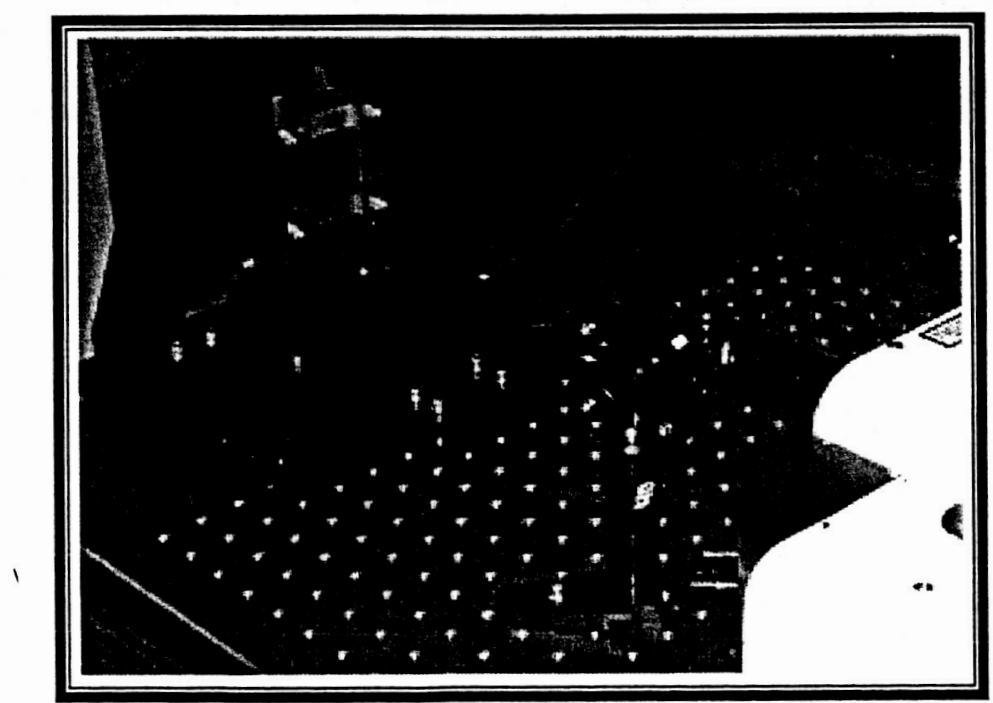

FIGURE 10. The optical set up for deformation measurements 


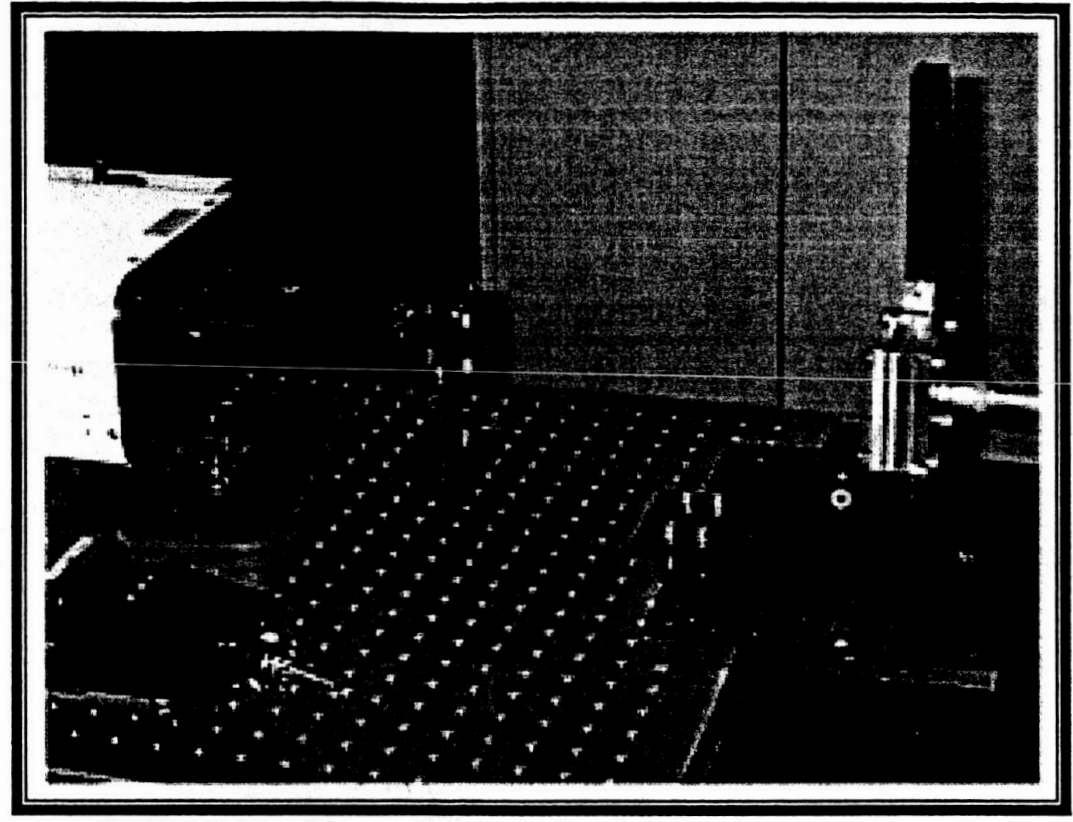

FIGURE 11. The optical set up for deformation measurements

The diverging lens is inside the tube in front of the interferometer. The quarter waveplate is moved from inside of the interferometer to the front of the diverging lens between the lens and the coupon. This modification is needed because the back reflection from the diverging lens has the same order of magnitude power as the returned test beam from the coupon. This back reflection washes out the deformation fringes.

An interferogram of the coupon is taken without any deformation and is stored as the baseline measurement; Figure 12.

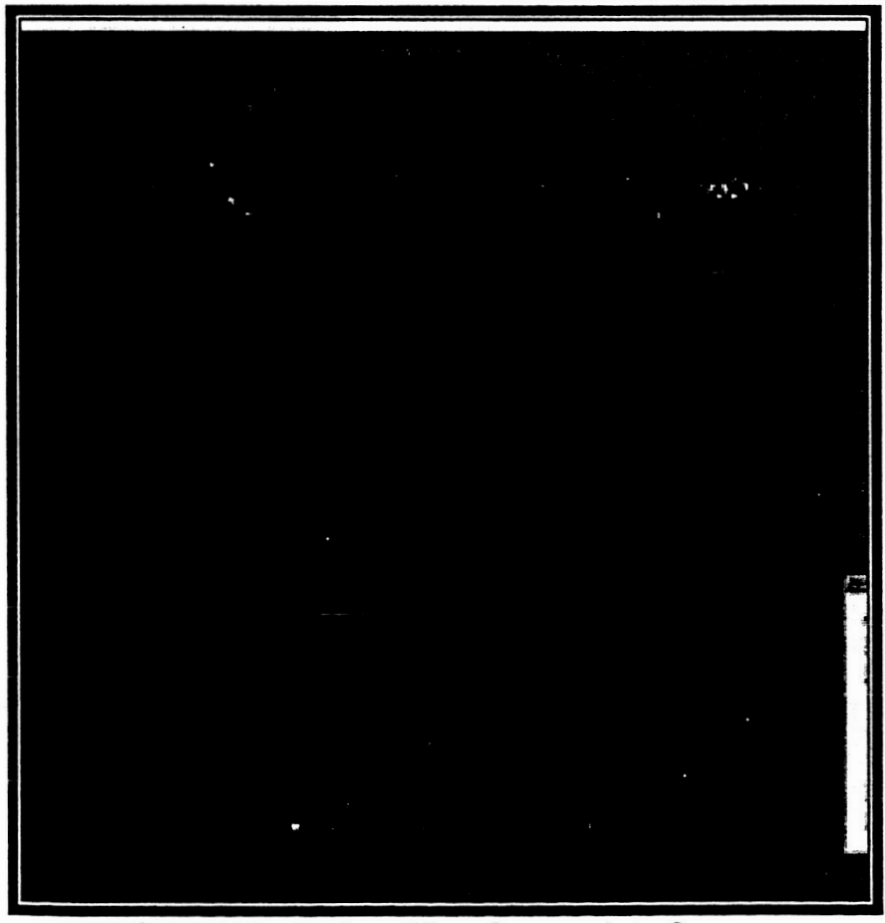

FIGURE 12 Carbon Fiber Coupon interferogram 
Initially the plunger gently touches the coupon. Plunger is moved by 0.5 micron. The smallest tick mark on the micrometer is 0.5 micron. Interferogram is acquired and subtracted from the baseline; Figure 13. Plunger is moved three and six tick marks; Figure 14, and Figure 15.

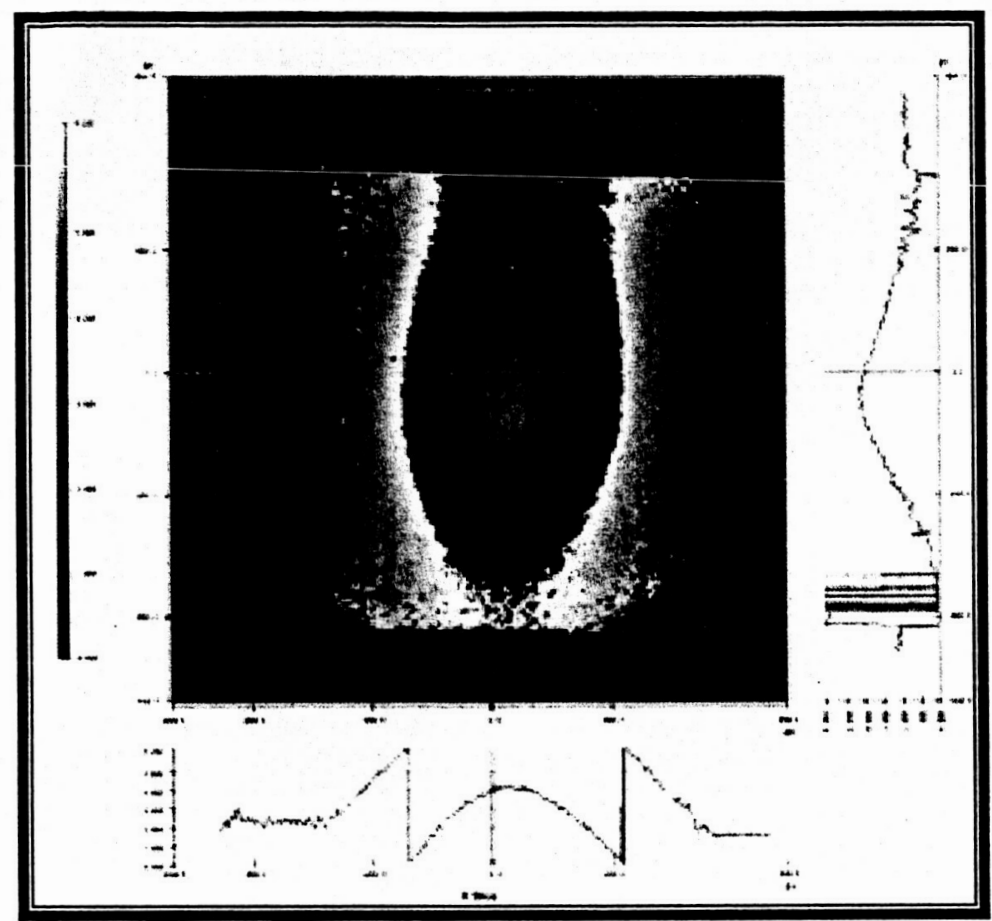

FIGURE 13 Fringes due to one tick mark motion of the micrometer

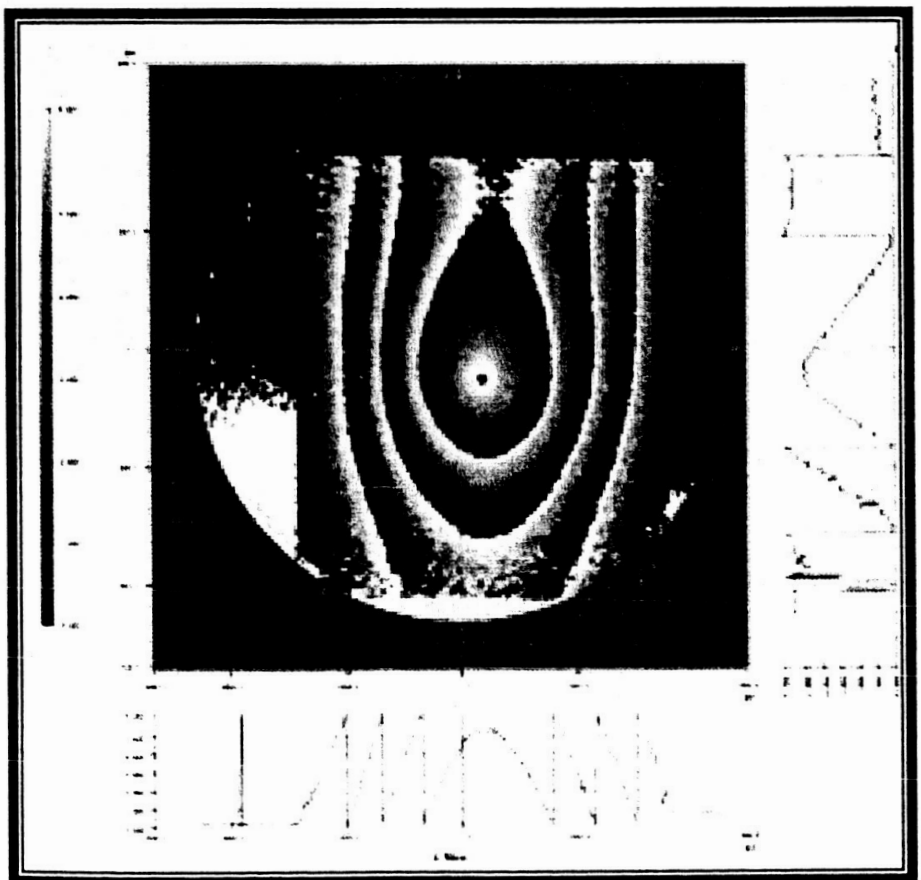

FIGURE 14 Fringes due to three tick marks motion of the micrometer 


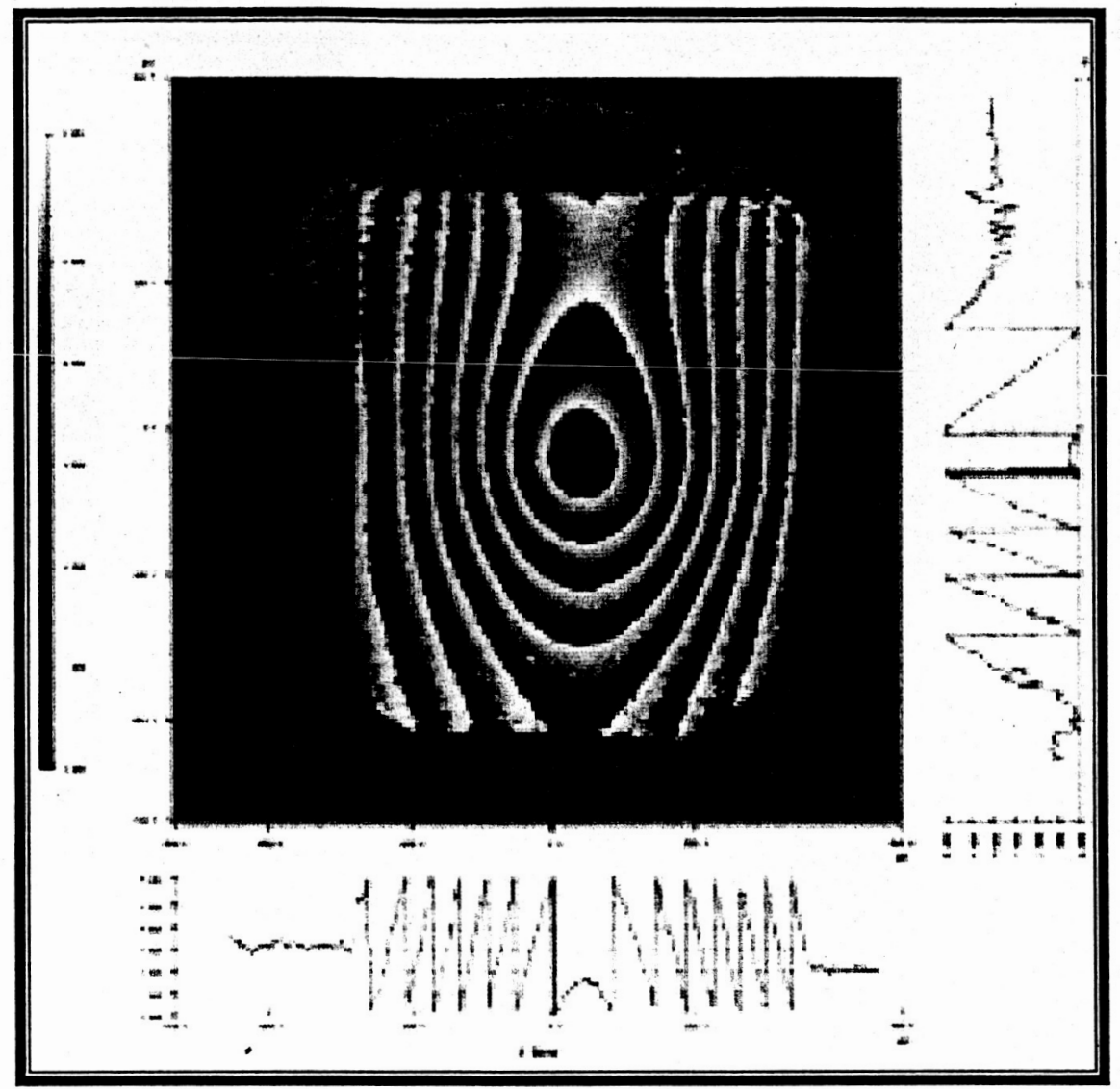

FIGURE 15 Fringes due to six tick marks motion of the micrometer

This step is repeated till the micrometer introduces 6.5 micron of deformation to the coupon; Figure 16. 


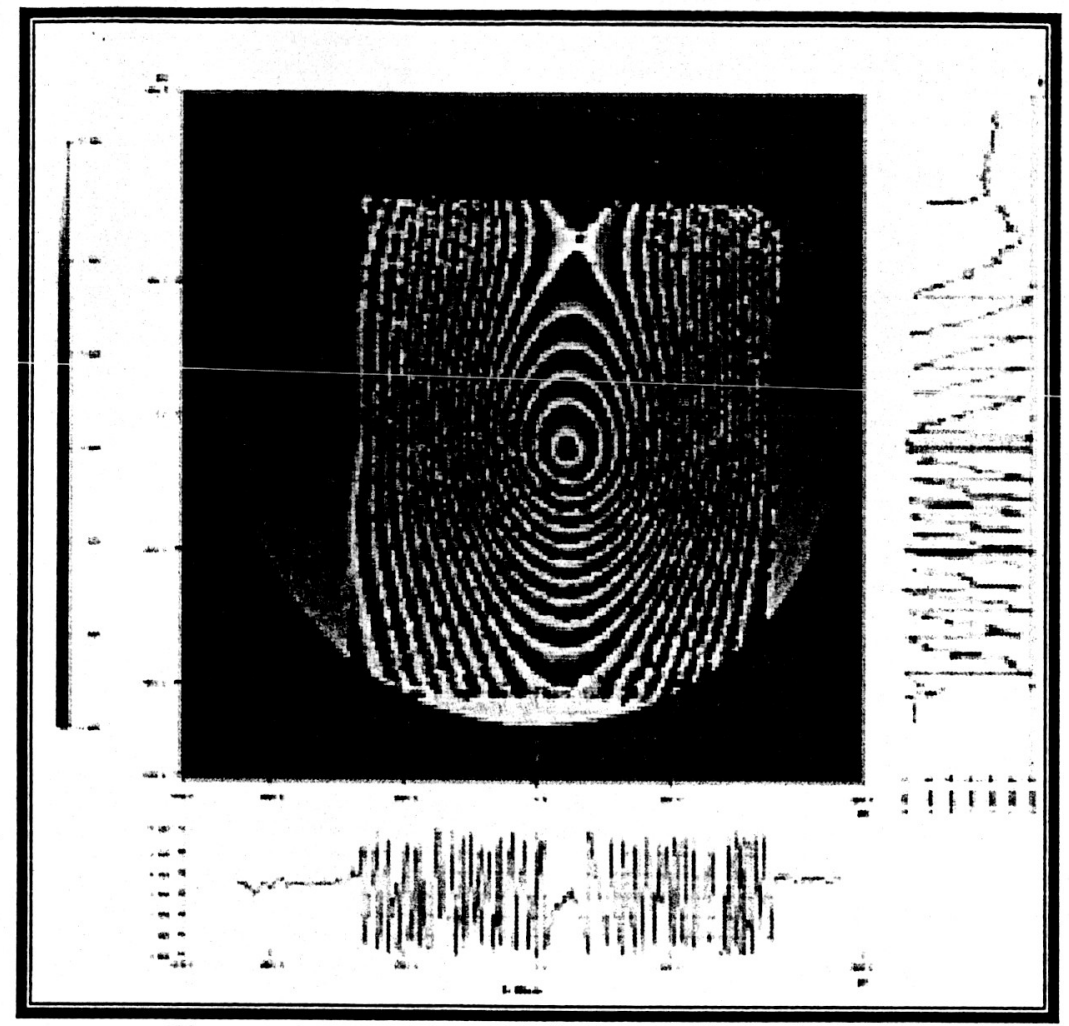

Figure 16. Fringes due to 6.5 microns of deformation

Next, the deformation data is unwrapped using the Flynn's algorithm and P-V surface deformation is plotted as function of the input deflection, Figure 17.

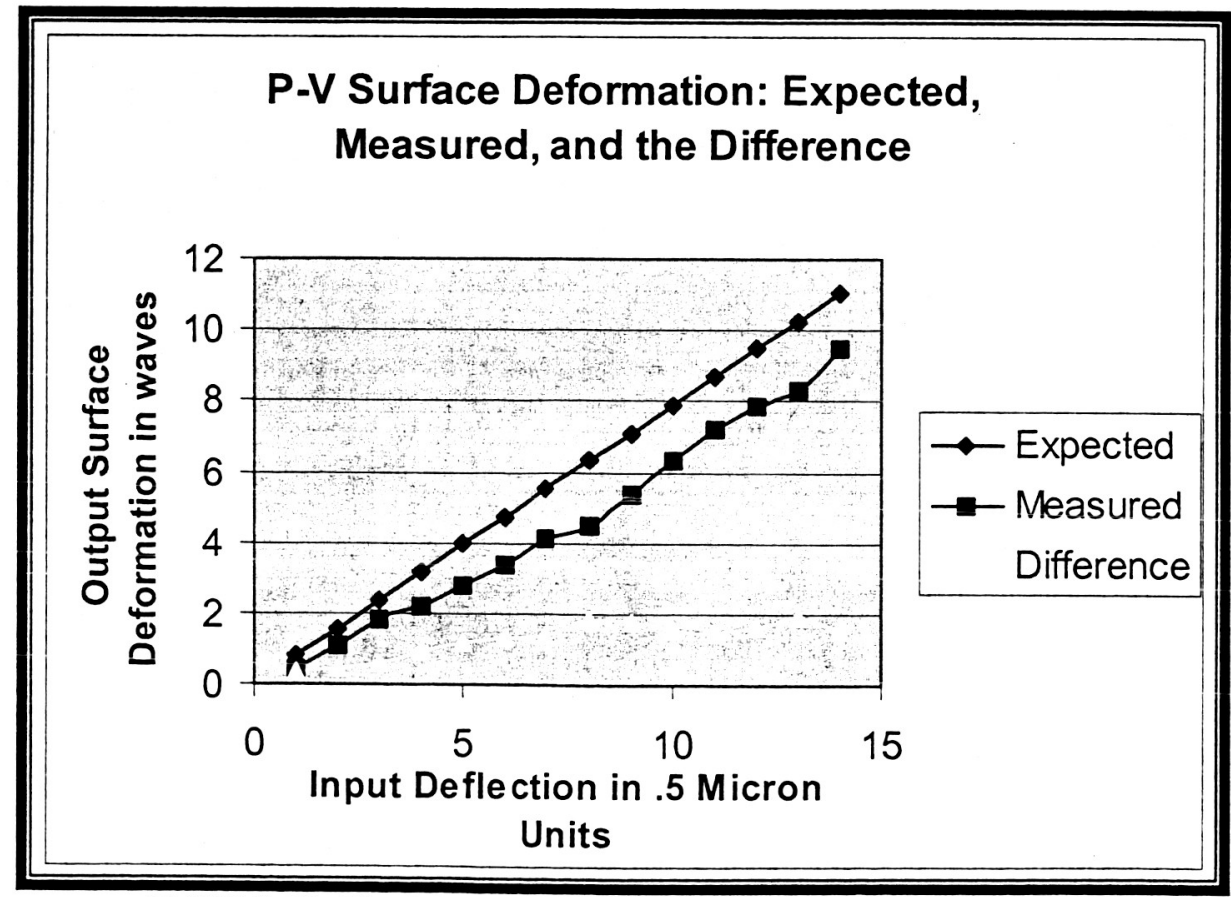

FIGURE 17. The P-V surface deformation as function of deflection 
The measured P-V deformation ( $)$ is below the expected values $(\vartheta)$ up to the $8^{\text {th }}$ tick marks after that, the two lines are almost parallel. The standard deviation for the parallel part, after the $8^{\text {th }}$ tick mark, is 0.1 micron, which is one fifth of a tick mark.

The measured $\mathrm{P}-\mathrm{V}$ is below expected because no preloading is done. The holder and sample move as a rigid body in the beginning, so the plunger motion deforms the coupon less than expected. Once the holder and the sample are not moving, then the motion of the plunger and the deformations are linear.

\section{CONCLUSION}

A vibration insensitive highly accurate measurement is the primary motivation to combine simultaneous phase shifting with speckle interferometry. A temporal phase shifted speckle interferometer takes about $70 \mathrm{~ms}$ to measure a phase deformation of a diffuse object given that $C C D$ integration time is less than the phase shifting. Simultaneous phase shifted speckle interferometer acquire the phase difference for the same object in 60 microsecond, three orders of the magnitude faster. The component that limits the processing time in simultaneous speckle phase shifting interferometry is the integration time of the $\mathrm{CCD}$. To minimize the integration time on the $\mathrm{CDD}$, irradiances need to be maximized. To maximize the reflected object irradiance, objects are painted with different kinds of white paints to increase the reflectivity of the surface. Space flight hardware such as Back Plane of PM or the Secondary Mirror Tower of the JWST cannot be painted. Increasing the illumination irradiance increases the reflected irradiance. To remedy the irradiance shortage, low power single mode (spatially and temporally) lasers in the speckle interferometers are replaced with high power single mode lasers. In addition, to increase the irradiances at the CCD, the aperture of the collecting optics can be increased. The size of the lens aperture is limited by the noise on the $\mathrm{CCD}[9]$.

\section{REFERENCES}

J.M. Burch, "Interferometry with scattered light", Optical Instruments and Techniques 1996 (Oriel Press, Newcastle upon Tyne, 213-229, 1970).

J.A. Leendertz, "Measurement of surface displacement by interference of speckle patterns", Optical Instruments and Techniques 1996 (Oriel Press, Newcastle upon Tyne, 256-264, 1970).

R.S. Sirohi and F.S. Chau, Optical Methods of Measurements Wholefield Techniques (Marcel Dekker, Inc. 1999).

R.S. Sirohi (Ed), Speckle Metrology (Marcel Dekker, NY 1993).

K. Creath, Phase - shifting speckle interferometry", Appl. Opt. 24, 3053-3058, 1985.

L. Yang and A. Ettemeyer, "Strain measurement by three - dimensional electronic speckle pattern interferometry: Potentials, limitation, and applications", Opt. Eng. 42(5), 1257-1266, 2003.

P.K. Rastogi, "Holography and speckle techniques applied to non destructive measurements and testing", Holography for the new millennium (H.J. Caufield and J. Riccobono (Eds), Springer-Verlag, NY Inc. 2001).

W. Steinchen, L.Yang, Digital Shearography Theory and Application of Digital Speckle Pattern Shearing Interferometry (SPIE Press 2003).

J.M. Huntley, "Random phase measurements errors in digital speckle interferometry", SPIE Vol. 2544, 246-257, 1995. 